\title{
The role of overexpressed DYRK1A protein in the early onset of neurofibrillary degeneration in Down syndrome
}

\author{
Jerzy Wegiel · Karol Dowjat · Wojciech Kaczmarski • Izabela Kuchna $\cdot$ Krzysztof Nowicki · Janusz Frackowiak • \\ Bozena Mazur Kolecka • Jarek Wegiel · Wayne P. Silverman • Barry Reisberg • Mony deLeon • \\ Thomas Wisniewski $\cdot$ Cheng-Xin Gong $\cdot$ Fei Liu $\cdot$ Tatyana Adayev $\cdot$ Mo-Chou Chen-Hwang $\cdot$ Yu-Wen Hwang
}

Received: 25 April 2008 / Revised: 18 July 2008 / Accepted: 21 July 2008 / Published online: 12 August 2008

(C) The Author(s) 2008. This article is published with open access at Springerlink.com

\begin{abstract}
The gene encoding the minibrain kinase/dualspecificity tyrosine phosphorylated and regulated kinase $1 \mathrm{~A}$ (DYRK1A) is located in the Down syndrome (DS) critical region of chromosome 21. The third copy of DYRK1A is believed to contribute to abnormal brain development in patients with DS. In vitro studies showing that DYRK1A phosphorylates tau protein suggest that this kinase is also involved in tau protein phosphorylation in the human brain and contributes to neurofibrillary degeneration, and that this contribution might be enhanced in patients with DS. To explore this hypothesis, the brain tissue from 57 subjects
\end{abstract}

J. Wegiel $(\bowtie) \cdot$ K. Dowjat · W. Kaczmarski $\cdot$ I. Kuchna ·

K. Nowicki · J. Frackowiak · B. Mazur Kolecka · J. Wegiel Department of Developmental Neurobiology,

NYS Institute for Basic Research in Developmental Disabilities, 1050 Forest Hill Road, Staten Island, NY 10314, USA

e-mail: J_Wegiel@msn.com

\section{W. P. Silverman}

Department of Psychiatry and Behavioral Medicine,

John Hopkins University Medical School, Baltimore, MD, USA

B. Reisberg $\cdot$ M. deLeon $\cdot$ T. Wisniewski

Department of Psychiatry,

New York University School of Medicine,

Silberstein Aging and Dementia Research and Treatment Center,

New York, NY, USA

C.-X. Gong · F. Liu

Department of Neurochemistry,

NYS Institute for Basic Research in Developmental Disabilities,

1050 Forest Hill Road, Staten Island, NY, USA

T. Adayev $\cdot$ M.-C. Chen-Hwang $\cdot$ Y.-W. Hwang

Department of Molecular Biology,

NYS Institute for Basic Research in Developmental Disabilities, 1050 Forest Hill Road, Staten Island, NY, USA including 16 control subjects, 21 patients with DS, and 20 patients with sporadic Alzheimer's disease (AD) was examined with two antibodies to the amino-terminus of DYRK1A (7F3 and G-19), as well as two polyclonal antibodies to its carboxy-terminus (X1079 and 324446). Western blots demonstrated higher levels of full-length DYRK1A in the brains of patients with DS when compared to control brains. Immunocytochemistry revealed that DYRK1A accumulates in neurofibrillary tangles (NFTs) in subjects with sporadic AD and in subjects with DS/AD. Overexpression of DYRK1A in patients with DS was associated with an increase in DYRK1A-positive NFTs in a gene dosage-dependent manner. Results support the hypothesis that overexpressed DYRK1A contributes to neurofibrillary degeneration in DS more significantly than in subjects with two copies of the DYRKIA gene and sporadic AD. Immunoreactivity with antibodies against DYRK1A not only in NFTs but also in granules in granulovacuolar degeneration and in corpora amylacea suggests that DYRK1A is involved in all three forms of degeneration and that overexpression of this kinase may contribute to the early onset of these pathologies in DS.

Keywords Minibrain kinase DYRK1A .

Neurofibrillary degeneration · Granulovacuolar degeneration - Corpora amylacea $\cdot$ Down syndrome Alzheimer's disease

\section{Introduction}

A broad spectrum of developmental and age-associated changes in patients with Down syndrome (DS) is considered to be a result of the overexpression of genes localized in the DS critical region of chromosome 21. The extra copy 
of the gene encoding amyloid precursor protein $(A P P)$ located on chromosome 21 appears to be the main cause of the early onset of brain amyloidosis- $\beta$ in patients with DS. Overexpression of APP has been associated with an increase in amyloid $\beta(\mathrm{A} \beta) 42$ level in the brain of fetuses with trisomy of chromosome 21 [64], the development of diffuse $\mathrm{A} \beta$-positive plaques in approximately $50 \%$ of individuals with DS younger than 30 years of age $[35,37,72$, 73], Alzheimer-type pathology in the majority of DS subjects older than 40 years of age $[55,69,73]$, and an elevated risk of Alzheimer's disease (AD)-associated dementia [24, 33, 41, 78].

While early brain amyloidosis- $\beta$ appears to be directly associated with an extra copy of the APP gene, the mechanism leading to neurofibrillary degeneration in patients with DS is unknown. Recent studies suggest that the Minibrain kinase/dual-specificity tyrosine phosphorylated and regulated kinase 1A (DYRK1A) gene, located in the critical region of chromosome $21[20,50,62]$ and overexpressed in DS [21, 27], may play a significant role not only in developmental brain defects associated with DS [46, 59] but also in neurofibrillary degeneration. The discovery that DYRK1A phosphorylates microtubule-associated protein tau at several phosphorylation sites and in cultured cells $[40,74]$ implicates DYRK1A in abnormal tau phosphorylation and neurofibrillary degeneration in patients with AD. The observation that $\mathrm{Thr}^{212}$ is phosphorylated in fetal tau and hyperphosphorylated in filamentous tau from subjects with AD [43] supports this hypothesis. Moreover, phosphorylation of $\mathrm{Thr}^{212}$ by DYRK1A stimulates in vitro the rate of phosphorylation of tau about eightfold through priming tau phosphorylation by glycogen synthase kinase 3 (GSK3) at $\operatorname{Ser}^{208}$ [74]. Phosphorylation of $\mathrm{Ser}^{208}$ has also been reported in tau in patients with AD [43]. DYRK1Ainduced tau phosphorylation inhibits tau activity to stimulate microtubule assembly and promotes its self-assembly into filaments [40]. These findings strengthen the position that DYRK1A may be involved in tau protein phosphorylation in the human brain and potentially in neurofibrillary degeneration.

The aim of this study was to explore the hypothesis that DYRK1A contributes to neurofibrillary degeneration in patients with sporadic $\mathrm{AD}$ and that this contribution is enhanced in DS subjects with trisomy of chromosome 21 and AD. Western blots demonstrated higher levels of DYRK1A in the brains of patients with DS, whereas immunocytochemistry with antibodies to DYRK1A showed the presence of this kinase in neurofibrillary tangles (NFTs) in patients with sporadic AD. A several-fold increase in the number of DYRK1A-positive NFTs in patients with DS/AD supports the hypothesis that DYRK1A contributes to the early onset of neurofibrillary degeneration in DS.

\section{Materials and methods}

Tissue for morphological studies

Brains of 57 subjects were examined, including 16 control subjects 32-92 years of age (12 males and 4 females), 21 subjects with DS from 38 to 72 years of age (12 males and 9 females), and 20 subjects with sporadic AD 73-97 years of age (11 males and 9 females). Functional assessment staging (FAST) [51] was used to evaluate the progression of the clinical course of sporadic AD. Control subjects were classified as FAST stages 1 and 2 (normal adult without functional decrement documented in available medical records, and normal aged adult with subjective deficit in word finding, respectively). At the time of their demise, three AD subjects were at FAST stage 3 (incipient AD with deficits noted in demanding employment settings); three subjects at FAST stage 4 (mild AD with deficits in performance of complex tasks of daily life); two subjects at FAST stage 5 (moderate AD manifested with deficient performance in choosing proper attire); four subjects at FAST stage 6 (moderately severe AD manifested with five substages, including decreased ability to dress properly, to handle mechanics of bathing and toileting, and finally with urinary and fecal incontinence), and eight subjects at FAST stage 7 (severe AD with several substages, including incipient averbalism, limitation of speech to a single word, and loss of ambulation). Patients were examined by clinicians in the Aging and Dementia Research Center at New York University Medical Center.

Fixation, processing, embedding, and neuropathological evaluation

One brain hemisphere from each subject was cut into $1-\mathrm{cm}$ thick frontal slabs. Diagnostic tissue samples were cut off and fixed in formalin for routine neuropathological examination, while the majority of tissue was frozen at $-80^{\circ} \mathrm{C}$ for biochemistry. Neuropathological evaluation was based on gross brain description and examination of $8-\mu \mathrm{m}$-thick sections from 16 brain regions. Alzheimer-type pathology was evaluated using criteria developed at a consensus conference organized by the National Institute on Aging and the Reagan Institute of the National Alzheimer Disease Association [44]. According to Braak and Braak criteria [5], all control subjects younger than 42 years of age were free of AD pathology. All older control subjects were affected with incipient neurofibrillary degeneration corresponding to Braak and Braak criteria limbic stage I. They were free of amyloid plaques. AD subjects represented a broad spectrum of neuropathological changes from Braak and Braak limbic stage II to neocortical stage VI. In the examined cohort, all subjects with DS older than 38 years of age were affected 
by both neurofibrillary degeneration and $\beta$-amyloidosis with neuritic plaques corresponding to Braak and Braak II-VI.

The other hemisphere was fixed in $10 \%$ buffered formalin for 6 weeks to several months and then dissected into 1-cm-thick frontal slabs. The tissue blocks were dehydrated in a graded series of ethanol: for 5 days, in $70 \%$ ethanol; then 2 days in $80 \%$ ethanol; and finally, 1 week in $96 \%$ ethanol. Dehydrated tissue was infiltrated with polyethylene glycol (PEG) 400 (Merck \#807 485) for 6 days (two changes of 3 days each at room temperature) and with PEG 1000 for another 6 days (two changes of 3 days each at $42^{\circ} \mathrm{C}$ ). Slabs were embedded in fresh PEG 1000 [25] and stored in $+4^{\circ} \mathrm{C}$. Tissue blocks were then cut at a temperature of $18^{\circ} \mathrm{C}$ into $50-\mu \mathrm{m}$-thick serial sections. They were stored in $70 \%$ ethyl alcohol at room temperature and used for immunocytochemical and morphometric studies.

The methods applied to this study were approved by the Institutional Review Board at the New York State Institute for Basic Research in Developmental Disabilities. All brain tissue samples were identified by case number and examined blind to clinical and demographic information.

Immunostaining

Serial sections stored in $70 \%$ ethyl alcohol were washed with phosphate buffer solution (PBS) and immunostained as free-floating sections. Several antibodies were used to detect DYRK1A (Table 1), including two antibodies to the amino-terminus of DYRK1A: monoclonal antibody (mAb) 7F3 (IBR) [70] and goat polyclonal antibody (pAb) Dyrk1A G-19 (Santa Cruz Biotechnology Inc.). The carboxy-terminus of DYRK1A was detected with sheep pAbs X1079 from Exalpha Biologicals and sheep antibody 324446 from Calbiochem. Abnormally phosphorylated tau protein of NFTs was detected with mAb Tau-1. Tau-1 recognizes an epitope between amino acids 189 and 207 of the human tau sequence [17]. To obtain optimum staining with Tau-1, sections were dephosphorylated with alkaline phosphatase prior to the application of primary antibody [19] (Sigma, Type VII-L, $400 \mu \mathrm{g} / \mathrm{ml}$ in PBS, pH 7.4). A $\beta$-positive plaques were immunodetected with mouse mAb $4 \mathrm{G} 8$ (NYS, IBR), which recognizes amino acids $17-24$ of the amyloid $\beta$-peptide [29]. To enhance the immunoreactivity of $\mathrm{A} \beta$, sections were treated with concentrated formic acid [31].

The endogenous peroxidase in the sections was blocked with $0.2 \%$ hydrogen peroxide in methanol. Non-specific bindings were blocked with $10 \%$ fetal bovine serum in PBS for $30 \mathrm{~min}$. The antibodies were diluted in $10 \%$ fetal bovine serum in PBS and were incubated with sections overnight at $4^{\circ} \mathrm{C}$. The sections were washed and treated for $30 \mathrm{~min}$ with either biotinylated sheep anti-mouse IgG antibody or 
biotinylated donkey anti-goat IgG antibody diluted 1:200. The sections were treated with an extravidin peroxidase conjugate $(1: 200)$ for $1 \mathrm{~h}$, and the product of reaction was visualized with diaminobenzidine $(0.5 \mathrm{mg} / \mathrm{ml}$ with $1.5 \%$ hydrogen peroxide in PBS). After immunostaining, the sections were lightly counterstained with cresyl violet.

To confirm the specificity of the immunostaining, the reaction was tested with antibodies not absorbed as well as those absorbed with appropriate peptides. For absorption, antibody at the dilution 1:50 or 1:75 (the dilution at which consistently positive results are achieved on sections) was combined with a fivefold (by weight) excess of blocking peptide. The mixture was incubated for $2 \mathrm{~h}$ at room temperature (or overnight at $4^{\circ} \mathrm{C}$ ) and used for immunocytochemical tests. The effect of tau protein dephosphorylation was examined on sections pretreated and those not treated with alkaline phosphatase.

\section{Co-localization of Mnb/Dyrk1A and NFTs by double} immunofluorescence

Fifty- $\mu \mathrm{m}$-thick brain sections were double-immunostained for DYRK1A using pAb G19, and for NFTs, using mAb Tau-1. Sections treated with alkaline phosphatase were incubated with G19 antibody, followed by a secondary donkey antiserum against goat IgG conjugated with biotin (Amersham), and then streptAvidin-FITC (Vector). The second primary antibody was mAb Tau- 1 . The reaction was detected with antibody made in sheep: $\mathrm{F}(\mathrm{ab})_{2}$ fragments against mouse IgG conjugated to Cy3 (Sigma). The absence of cross-reactions was confirmed as described earlier [15, 16]. Images were generated using a Nikon Eclipse E800 microscope and the Nikon PCM 2000 dual-laser scanning confocal microscopy system and with the C-Imaging-SIMPLE32 ${ }^{\mathrm{TM}}$ image analysis system (Compix Inc.).

\section{Detection of DYRK1A using Western blotting}

Frozen tissue samples from the cerebral frontal cortex of five control subjects 31-71 years of age, 12 DS subjects from 28 to 63 years of age, and four subjects with AD from 71 to 85 years of age were used for the detection of DYRK1A using Western blotting. Tissue was mortarground in liquid nitrogen. $0.1 \mathrm{~g}$ of powdered brain tissue was lysed in $1 \mathrm{ml}$ of $6 \mathrm{M}$ guanidine chloride buffer $(6 \mathrm{M}$ $\mathrm{GuHCl}, 10 \mathrm{mM} \mathrm{NaH} \mathrm{PO}_{4}, 10 \mathrm{mM}$ Tris, $20 \mathrm{mM}$ imidazole, $\mathrm{pH} 8$ ) and sonicated. The protein concentration was determined by bicinchoninic acid assay (BCA) (Pierce Rockford, IL, USA). The total amount of protein extracted from each brain sample was calculated, and volumes corresponding to $4 \mathrm{mg}$ of protein were mixed with $50 \mu \mathrm{l}$ of Ni-Sepharose High Performance (Amersham) slurry and incubated for $2 \mathrm{~h}$ at $4^{\circ} \mathrm{C}$ with constant rotation. Ni-Sepharose beads were then washed three times with $1 \mathrm{ml} 8 \mathrm{M}$ urea buffer ( $8 \mathrm{M}$ urea, $100 \mathrm{mM} \mathrm{NaH} \mathrm{PO}_{4}, 10 \mathrm{mM}$ Tris, $\mathrm{pH} 5.9$ ) for each wash. Elution was carried out by boiling for $2 \mathrm{~min}$ in $50 \mu \mathrm{l}$ of $1 \times$ tricine sample buffer (BioRad) with $50 \mathrm{mM}$ EDTA. Ten microliter of each eluate was electrophoresed on $8 \%$ tricine SDS-polyacrylamide gel and transferred onto PVDF membrane. The membranes were blocked in $5 \%$ non-fat milk and incubated overnight with the appropriate dilution of monoclonal 7F3 [70] and goat polyclonal G19 (Santa Cruz Biotech, Inc.) antibodies. After incubation with alkaline phosphatase-conjugated secondary antibody (Amersham), the membranes were developed by color reaction using BCIP/NBT as a substrate. The immunoreactive bands on digitalized images were densitometrically quantified using the 1D Scan EX3.1 software program (Scanalytics Corp., Rockville, MD, USA).

\section{Morphometry}

On consecutive sections from the brains of subjects with $\mathrm{DS} / \mathrm{AD}$ and sporadic $\mathrm{AD}$, the numerical density $\left(\mathrm{n} / \mathrm{mm}^{3}\right)$ of neurons stained with cresyl violet $(\mathrm{CV})$, and of neurons with NFTs immunostained with antibodies Tau-1 and G-19 was evaluated. NFTs were counted in the four layers of the entorhinal cortex known to be affected early and severely with neurofibrillary degeneration (transentorhinal/entorhinal stage of neurofibrillary degeneration) [5], including layers II (islands of stellate cells), III, V, and VI. Sectors CA1 and CA4 of the cornu Ammonis and the pyramidal layer in the subiculum proper were selected as representative for the limbic stage of neurofibrillary degeneration. Neurons with NFTs were counted in a frame of $0.0035 \mathrm{~mm}^{2}$ in the entorhinal cortex and in a frame of $0.007 \mathrm{~mm}^{2}$ in three subdivisions of the hippocampal formation. The depth of the examined dissector was $30 \mu \mathrm{m}$. Based only on neurons containing Tau-1-positive NFTs, the proportion of neurons with G-19-positive NFTs was calculated. NFTs were counted in three histological sections representing the frontal, middle, and caudal portions of the entorhinal cortex and hippocampal formation. Twenty-five test areas were evaluated for each examined subregion at a final magnification of $3,100 \times$.

\section{Results}

All samples were subjected to partial purification by immobilized metal $\left(\mathrm{Ni}^{2+}\right)$ ion affinity chromatography (IMAC). DYRK1A binds with high affinity to nickel ions chelated to sepharose matrix through the histidine-rich domain in the C-terminal part of the molecule $[20,23]$. The samples were made out of pools of cerebral frontal cortex tissue of five control, 12 DS, and four AD subjects. Figure 1 shows the 


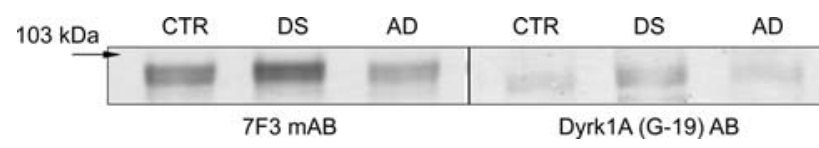

Fig. 1 Representative Western blot of Ni-affinity purified Dyrk1A from the pools of the cerebral frontal cortex tissue of control (CTR five cases; 31-71 years), Down syndrome (DS 12 cases; 28-63 years), and Alzheimer disease ( $A D$ four cases; 71-85 years) subjects. Mixing equal amounts of powdered brain tissue from each case made pools, which were then processed for Western blotting and densitometric quantification. Equal loads of two identical sets of samples were separated on $8 \%$ tricine SDS-polyacrylamide gel and immunoblotted with a 1:5,000 dilution of monoclonal 7F3 antibody at a 1:10,000 dilution of polyclonal G19 antibody. The blot was developed by color reaction using AP-conjugated secondary antibodies and BCIP/NBP substrate. The density of immunoreactive bands on digitalized images was measured by the 1D Scan EX3.1 software program. The value of the DS:CTR ratio was the mean \pm SD of three independent Western blots. Densitometry of control and DS samples revealed an enhancement of DYRK1A levels in the DS pool by a factor of 1.47 for 7F3, and 1.9 for G19 antibody. Both antibodies revealed less DYRK1A in the AD pool when compared to controls

immunoblot of IMAC-purified samples representing each pool, developed with monoclonal 7F3 and polyclonal G19 antibodies, both directed against the $\mathrm{N}$-terminus of DYRK1A protein. In all three pools, 7F3 and G19 antibody detected, with similar relative intensity, two closely spaced bands with the approximate molecular weights of 97 and $94 \mathrm{kDa}$. Densitometrical quantification of immunoreactive bands revealed an enhancement of DYRK1A levels in the DS pool by the factor of 1.47 and 1.9 for $7 F 3$ and G19 antibody, respectively. When compared with control subjects, the AD pool showed visibly less DYRK1A by both antibodies. The data shown in Fig. 1 are part of our published study [13] in which cases comprising each pool were tested and evaluated individually. In the course of this study, we measured the levels of DYRK1A in six brain structures and found a statistically significant $(P<0.05)$ increase in the DS group. The overall increase calculated for all six structures was 1.43 -fold, very close to the predicted gene dosage value of 1.5 .

Immunodetection of DYRK1A in neurons free of neurofibrillary degeneration

Immunostaining with mAb 7F3 revealed Mnbk/Dyrk1A in the nucleus and cytoplasm of neurons, including neuronal processes and synapses. However, the reaction varied substantially, ranging from heavy granular staining in the cell nucleus and cytoplasm to just traces or even a total lack of reaction product. The immunoreactivity was neuron typeand brain structure-specific. Cytoplasmic immunoreactivity with $\mathrm{mAb} 7 \mathrm{~F} 3$ in normal brain was characterized in our previous article [70]. Antibodies to DYRK1A G-19, $\mathrm{X} 1076$, and 324446 also immunolabeled neurons in the brains of controls, patients with DS, and patients with sporadic AD. Immunoreactivity was observed mainly in the cell body, but some reactivity was present in apical dendrites, especially in sections stained with mAb 7F3 (not shown) and pAb G-19 (Fig. 2a-c).

\section{DYRK1A immunoreactivity in NFTs}

Antibody Tau-1 to tau protein and three antibodies to DYRK1A (G-19, X1079, and 324446) immunolabeled neurons in different stages of NFT formation (Fig. 2d-i). In the early stage of neurofibrillary degeneration, formation of NFTs was associated with enlargement of the cell body and deformation and displacement of the cell nucleus to the cell periphery. In this stage, the entire cytoplasm, including NFTs, showed weak to moderate immunoreactivity to DYRK1A (Fig. 2d-f). The progression of changes was associated with a more selective enhancement of reaction in bundles of cytoplasmic fibers with the morphology of NFTs and the proximal portion of dendrites, including apical dendrites of pyramidal neurons (Fig. $2 \mathrm{~g}-\mathrm{i}$ ). In contrast to the common and strong pAb G-19 immunoreactivity of intraneuronal NFTs, and the weaker immunoreactivity with pAbs to the C-terminus of DYRK1A (X1079 and 324448), NFTs released from dead neurons (ghost tangles) were always immunonegative (Fig. 2j-1). Mab 7F3 did not detect intracellular or extracellular NFTs (ghost tangles, Table 2).

Immunostaining with pAb G-19 revealed numerous DYRK1A-positive NFTs in the entorhinal cortex, limbic structures, and isocortex in subjects with DS, but many fewer in patients with sporadic AD (Fig. 3).

The appearance of Tau-1 immunoreactive NFTs in neuronal bodies was associated with the appearance of Tau-1-positive deposits in dendrites (neuropil threads). The increase in the number of Tau-1-positive neuronal bodies was paralleled with an increase in the number of Tau-1immunopositive neuropil threads in DS/AD and sporadic AD. However, in sections stained with antibodies G-19, X1079, and 324448, immunolabeling of NFTs in cell bodies was observed in the almost total absence of immunoreaction in neuropil threads for both groups (Fig. 3). The increase in the number of DYRK1A-positive NFTs in persons with DS and severe Alzheimer-type pathology was not associated with an increase in the number of immunopositive neuropil threads.

Colocalization of abnormally phosphorylated tau and DYRK1A in NFTs

Intraneuronal co-localization of DYRK1A and NFTs was confirmed by double-immunofluorescence and confocal microscopy. For Tau-1-negative neurons, the staining for DYRK1A was granular and the product of immunoreactivity 
Fig. 2 Antibodies to the N-terminal $(G-19)$ and the C-terminal portion (X1079 and 324446) of DYRK1A reveal immunopositive material in the cytoplasm in neurons in CA1 (a), the nucleus basalis of Meynert (b), and CA4 (c) in a 31-year-old control subject (arrowheads). Early stage of neurofibrillary degeneration was associated with an increase in the size of neuronal body filled with G-19-, X1079-, and 324446-positive NFTs; displacement of the nucleus to the cell periphery; and diffuse cytoplasmic immunoreactivity in cell cytoplasm (arrowheads CA4 sector in a 52-year-old subject with DS/AD; $\mathbf{d}-\mathbf{f}$ ). In the advanced stage of NFT formation, immunopositive material marks mainly bundles of NFTs (arrowheads CA1 sector in a 52-yearold subject with DS/AD: $\mathbf{g - i})$.

However, ghost tangles (arrows) stained with these three antibodies (CA1 sector in a 52-year-old subject with DS/AD; $\mathbf{j}-\mathbf{l})$.

Magnification: a $\times 390$;

b-I $\times 690$ are immunonegative when
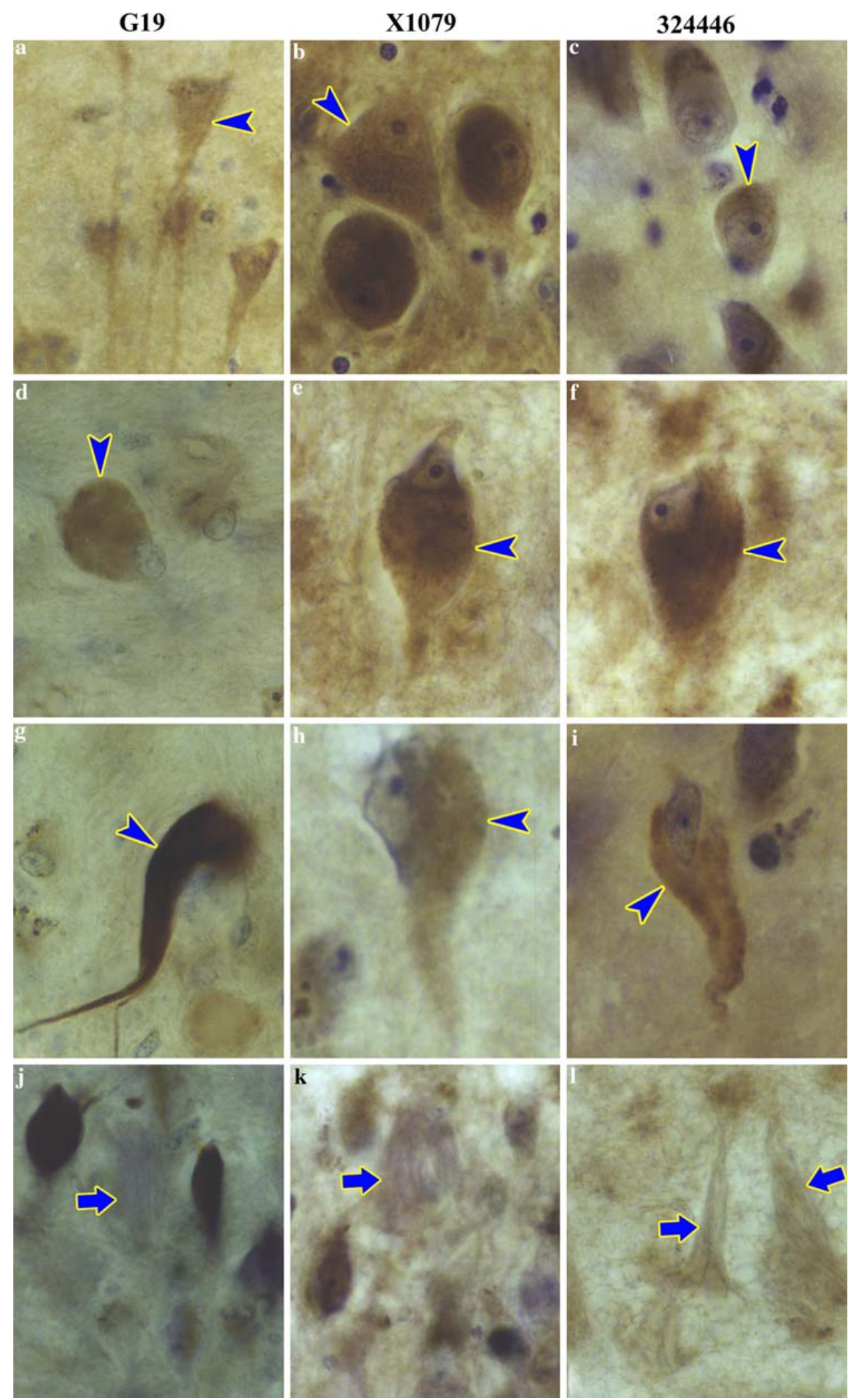

was evenly distributed in the cytoplasm in both the neuronal body and cell nucleus (Fig. 4a; and higher magnification, Fig. 4aa). In some neurons, the accumulation of abnormally phosphorylated tau in the early stage of neurofibrillary degen- eration (pretangle stage) was associated with the formation of numerous Tau-1-immunopositive cytoplasmic granules in the cell body and dendritic tree (Fig. 4b, bb). Some Tau-1-positive deposits were co-localized with G-19-immunofluorescent 
Table 2 Immunoreactivity in neurons, glial cells, and corpora amylacea with antibodies to DYRK1A

\begin{tabular}{|c|c|c|c|c|}
\hline \multirow[t]{2}{*}{ Structures } & \multicolumn{4}{|l|}{ Antibody } \\
\hline & 7F3 N-terminus & G-19 N-terminus & X1079 C-terminus & 324446 C-terminus \\
\hline \multicolumn{5}{|l|}{ Neuron } \\
\hline Nucleus & +++ & + & + & + \\
\hline Cytoplasm & +++ & ++ & ++ & ++ \\
\hline NFTs & - & ++++ & ++ & ++ \\
\hline Neuropil threads & - & \pm & \pm & \pm \\
\hline Ghost tangles & - & - & - & - \\
\hline Dystrophic processes in amyloid plaques & + & + & + & + \\
\hline GVD (cores) & - & - & ++ & ++ \\
\hline \multicolumn{5}{|l|}{ Astrocyte } \\
\hline Nucleus & - & - & - & - \\
\hline Cytoplasm & ++ & ++ & ++ & ++ \\
\hline \multicolumn{5}{|l|}{ Microglia and oligodendrocytes } \\
\hline Nucleus, cytoplasm & - & - & - & - \\
\hline Corpora amylacea & +++ & ++ & ++ & ++ \\
\hline
\end{tabular}

NFTs neurofibrillary tangles, GVD granulovacuolar degeneration

material, but some were not (Fig. 4c, cc). In spite of the strong immunoreactivity of DYRK1A in both the cell body and processes, co-localization of Tau-1- and G-19-positive material was limited almost exclusively to the cell body. The progression of neurofibrillary changes was reflected in an increase in the amount of Tau-1-positive material and the formation of bundles of NFTs immunoreactive only with Tau-1 or both Tau-1 and G-19 (Fig. 4d-f). In some neurons, the portion of bundles of NFTs was immunoreactive with Tau-1 or G-19 only, or with both antibodies (Fig. 4g-i). Tau-1-positive neuropil threads were almost always G-19-negative. In contrast to the uniform distribution of a fine granular product of reaction with pAb G-19 observed in normal neurons, G-19-immunopositive material formed several clusters of rough granules in astrocytes (Fig. 4d, f).

DYRK1A-immunoreactive NFTs in morphometric study

Figure 5 illustrates the result of the immunocytochemistrybased quantitative evaluation of G-19-positive NFTs in subjects with DS/AD and sporadic AD. In subjects with sporadic AD, the percentage of G-19-positive NFTs was structure-specific: $10 \%, 16 \%$, and $14 \%$ in layers II, III, and $\mathrm{V}$ in the entorhinal cortex, respectively; $24 \%$ in the pyramidal layer in the CA1 sector; but only $7 \%$ in the CA4 sector and $12 \%$ in the pyramidal layer of the subiculum proper. In subjects with DS/AD, the numerical density of G-19-positive NFTs in entorhinal cortex layers II, III, and V was increased by about $3.7 \times, 2.0 \times$, and $3.0 \times$, respectively. In the CA1 and CA4 sectors, their numbers were $1.5 \times$ and $3.6 \times$ higher, respectively, and in the subiculum, the number was increased by $2.6 \times$.
Neuronal loss and neurofibrillary degeneration in the second layer of the entorhinal cortex (EC) of subjects with DS/AD and sporadic AD is illustrated in Fig. 6. The similar age at the onset of AD pathology in almost all DS subjects results in the rather uniform neuronal loss in the second layer of the EC of DS subjects and the correlation between the numerical density of $\mathrm{CV}$-positive neurons and age $(r=0.75)$. The neuronal loss is paralleled by a decrease in the numerical density of Tau-1-positive NFTs $(r=0.65)$. The immunoreactivity of NFTs with G19 in all DS subjects, including the youngest in the examined cohort (38 years), suggests that DYRK1A contributes to the early onset of neurofibrillary degeneration in DS subjects. The increase in the percentage of G19-positive NFTs (considering Tau-1-positive neurons as $100 \%)$ with age $(r=0.51)$ suggests that DYRK1A contribution to neurofibrillary degeneration in DS increases with age. In three DS subjects, 58, 63, and 67 years, almost all tau-positive NFTs were G19-positive.

In the $\mathrm{AD}$ cohort, the numerical density of CV-positive neurons in the second layer of the EC decreases from $30,000-40,000 \mathrm{~mm}^{-3}$ in the early stages to about 15,000 in the late stages (Fig. 7b-f). In contrast to DS, sporadic AD starts at a different age, and the correlation between the number of neurons and age is not significant $(r=0.29)$, but the number of neurons correlates with FAST stage $(r=-0.87)$. The decrease in the numerical density of Tau-1-positive neurons correlates with the stage of $\mathrm{AD}(r=-0.76)$, but not with age $(r=0.22)$. In sporadic AD, G19-positive neurons represent a low percentage of Tau-1-positive NFTs. They were not found in eight subjects with sporadic AD. In six subjects, the 
Fig. 3 In a 67-year-old subject with DS, the presence of numerous Tau-1-immunoreactive NFTs in neuronal bodies and apical dendrites (arrows) is paralleled with the appearance of numerous Tau-1-immunopositive neuropil threads (arrowheads) in the amygdala (AMY), the second layer of the entorhinal cortex $(E C)$, and the pyramidal layer in the cornu Ammonis (CA1). In a subject with sporadic $\mathrm{AD}$ and moderately severe clinical pathology at the time of demise (FAST $6 d$ ), only a few NFTs (arrow) are G-19-positive. In contrast to Tau-1, which stains numerous neuropil threads, antibody G-19 does not detect neuropil threads. Magnification $\mathbf{a}-\mathbf{c} \times 55, \mathbf{d}-\mathbf{l} \times 430$

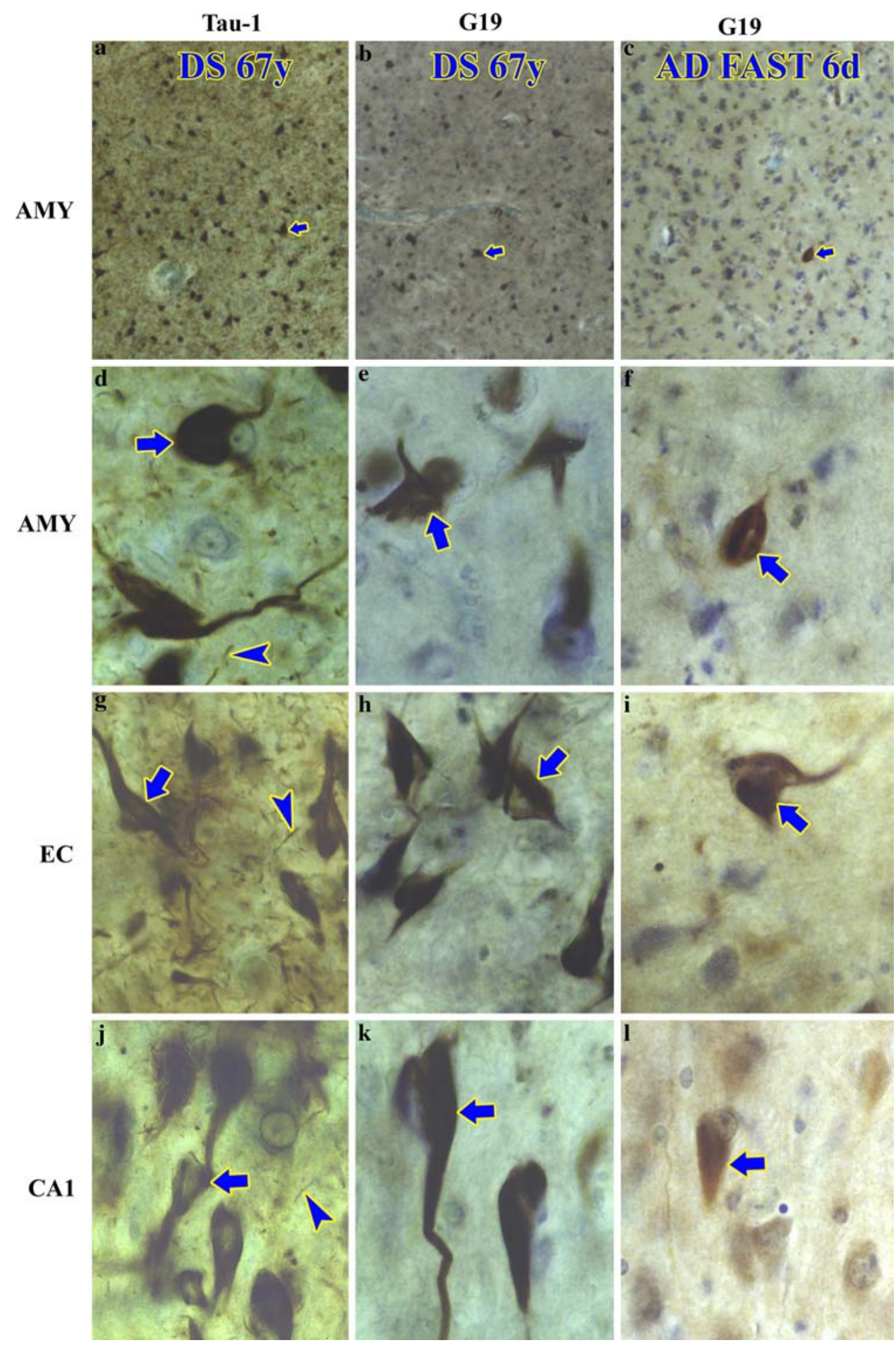

number was less than $10 \%$. In only six AD subjects, it varied from 25 to $67 \%$. In contrast to DS, in sporadic $\mathrm{AD}$, in the later stages of disease (FAST stages 6 and 7), the number of G19-positive NFTs was low, or they were not found.

Dephosphorylation with alkaline phosphatase did not change the pattern of DYRK1A distribution in NFTs (not shown) in the brains of people diagnosed with DS/AD or sporadic AD.

DYRK1A immunoreactivity in astrocytes

Two types of reaction were observed in astrocytes. In the majority of the subjects with DS, almost all astrocytes 
Fig. 4 Section from the cornu Ammonis of the 67-year-old DS subject immunostained with pAb G-19 (green color) and mAb Tau-1 (red color) and analyzed in confocal microscope.

Fine-granular, evenly distributed immunoreactivity is detected with pAb G-19 in the body of two neurons marked with arrowheads (a, aa higher magnification of one of these neurons). One neuron contains roughgranular deposits of Tau-1positive material (arrow) corresponding to an early stage of neurofibrillary degeneration (b, bb). Yellow color corresponds to partial co-localization of the product of reaction for DYRK1A and abnormally phosphorylated tau protein $(\mathbf{c}, \mathbf{c c})$. d-f A normal neuron positive for DYRK1A and negative for Tau-1 (1); a neuron with NFT immunoreactive with Tau- 1 only (2); and a neuron with NFT immunoreactive with both antibodies (3; G-19, green; Tau-1, red; co-localization, yellow). The cytoplasm of astrocytes is filled with clusters of G-19positive rough granules (arrowheads). A neuron in the late stage of neurofibrillary degeneration (g-i, arrowhead) contains some bundles of NFTs immunoreactive with both G-19 and Tau-1 (yellow). Magnification $\mathbf{a}-\mathbf{c}$ and $\mathbf{d}-\mathbf{f} \times 410$, aa-cc $\times 1,430, \mathbf{g}-\mathbf{i} \times 1,025$
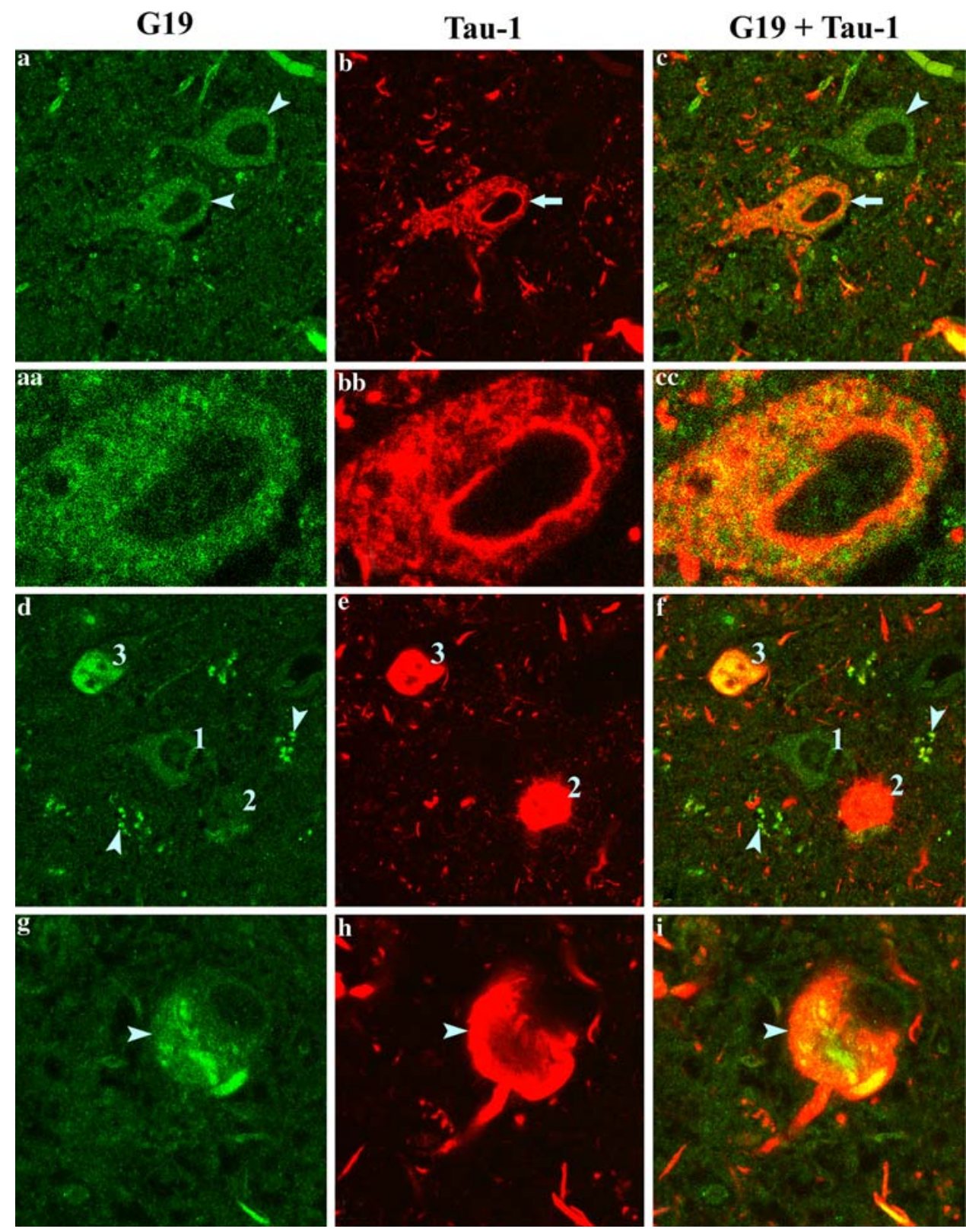

contained a variable amount of clusters of rough granules that were 7F3- and G-19-positive but Tau-1-negative. Immunoreactivity with X1079 and 324448 was less consistent and usually weaker than immunolabeling with 7F3 and G19. In patients with sporadic AD, granular staining in astrocytes was less pronounced, but astrocytes in the subependymal zone and in the molecular layer of allocortex and isocortex often showed mild to strong diffuse staining both in the astrocyte body and the processes. This pattern of staining was especially common and strong in astrocytes in neuritic plaque corona (Fig. $7 \mathrm{a}-\mathrm{c}$ ). Immunoreactivity with 7F3, G-19, X1079, and 324448 was undetectable in microglial cells and oligodendrocytes.

\section{DYRK1A immunoreactivity in neuritic plaques}

All subjects with DS/AD older than 38 years of age and all patients with sporadic AD were affected by amyloidosis, with numerous neuritic plaques detected with mAb 4G8 in both cortical and subcortical gray matter (not shown). In a large proportion of the neuritic plaques detected with $\mathrm{mAb}$ 4G8, mAb Tau-1-labeled numerous swollen dystrophic neuronal processes containing abnormally phosphorylated tau. Mild/moderate reactions with G-19, X1079, and 324448 were observed in about $20 \%$ of these plaques in areas corresponding to dystrophic neuronal processes (Fig. 7d-f). 


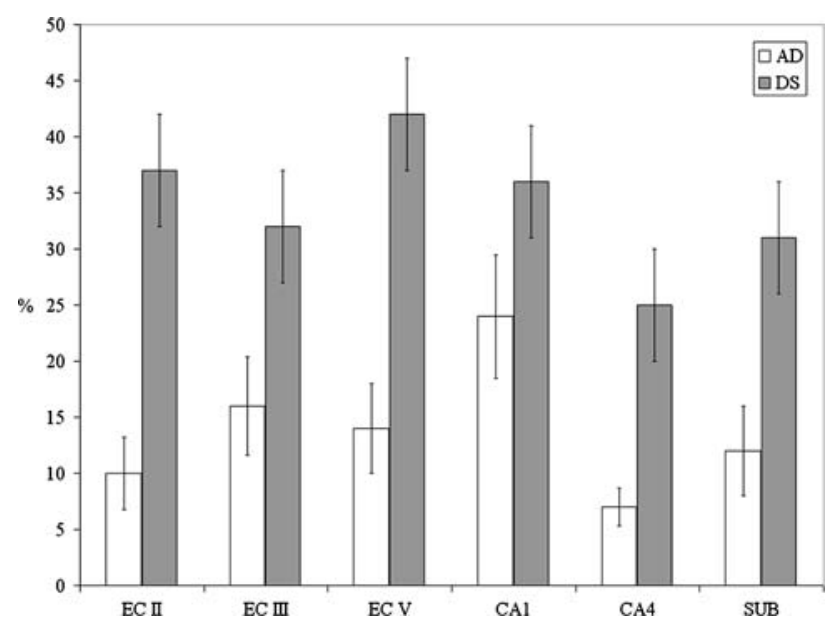

Fig. 5 Graph illustrates a several-fold higher percentage of G-19-positive NFTs (when the number of Tau-1-positive NFTs is considered $100 \%$ ) in the entorhinal cortex $(E C)$ layers II, III, and V, in the $C A I$ and $C A 4$, and in the subiculum proper $(S U B)$ in the brains of persons with DS compared to subjects with sporadic $A D$

Granulovacuolar degeneration and corpora amylacea

In subjects with $\mathrm{DS} / \mathrm{AD}$ and sporadic $\mathrm{AD}$, numerous neurons in the pyramidal layer of the cornu Ammonis were affected with granulovacuolar degeneration (GVD). The cell body contained several large vacuoles, each with a dense core. The number of affected neurons was increased in the middle stages of AD pathology. However, in subjects with severe AD, loss of neurons was associated with a reduction in the number of neurons with GVD. In the cornu Ammonis, immunostaining with C-terminal antibodies $\mathrm{X} 1079$ and 324448 revealed moderate to strong reactions in the cores in cytoplasmic vacuoles of neurons affected with GVD; however, reactions with N-terminal antibodies 7F3 and G-19 were undetectable (Fig. $7 \mathrm{~g}-\mathrm{i}$ ).

Corpora amylacea were a common finding in control subjects as well as in subjects with sporadic AD and DS/AD. They were strongly immunopositive in sections stained with 7F3 and moderately immunopositive in sections stained with G-19, X1079, and 324448 (Fig. 7j-1). A strong reaction was present not only in large corpora amylacea in the subpial, subependymal, and perivascular location but also in tiny corpora amylacea dispersed in the cortex and subcortical structures undetectable with $\mathrm{CV}$.

\section{Discussion}

Recent studies indicate that DYRK1A is involved in several molecular mechanisms leading to tau phosphorylation and that overexpression of DYRK1A in patients with trisomy of chromosome 21 and DS may contribute to the early onset of neurofibrillary degeneration. DYRK1A is present in the neuronal cytoplasm and nucleus [14, 70] and has been reported to phosphorylate both cytoplasmic and nuclear substrates [30, 40, 53, 74], suggesting that the subcellular localization of the protein reflects the cytoplasmic and nuclear pathway leading to neurofibrillary degeneration.

Cytoplasmic pathway

DYRK1A phosphorylates the human microtubule-associated protein tau at $\mathrm{Thr}^{212}$ in vitro, a residue that is hyperphosphorylated in AD and tauopathies, including Pick disease [14, 74], and permits phosphorylation of $\operatorname{Ser}^{208}$ by glycogen synthase kinase 3 [74]. Ferrer et al. [14] observed modifications in the expression of the constitutive cytoplasmic and nuclear DYRK1A in neurodegenerative diseases associated with tau phosphorylation including DS, AD, and Pick disease. However, they could not find any evidence that DYRK1A is directly involved in neurofibrillary degeneration.

Application of two antibodies to the N-terminal portion of DYRK1A, 7F3 [70] and G-19, and of two antibodies to the C-terminal portion of DYRK1A, X1076 and 324446, shows that the distribution of DYRK1A in the normal human brain undergoes modifications associated with neurofibrillary degeneration in DS/AD and sporadic AD. In control brains, all four antibodies reveal immunoreactivity in neurons, both in the cell body and the processes. A stronger reaction is observed in sections stained with antibodies to the $\mathrm{N}$-terminal compared to the $\mathrm{C}$-terminal portion of DYRK1A. DYRK1A contains a nuclear targeting sequence and is distantly related to the mitogen-activated protein kinase family [27]. Intranuclear localization of DYRK1A was shown in human neurons with mAb 7F3 [70], but three antisera, including G-19, detect less immunoreactivity in the cell nucleus.

Our study offers evidence that DYRK1A is directly involved in neurofibrillary degeneration. Antiserum G-19 shows a strong reaction in the majority of NFTs in patients with DS, and antisera X1079 and 324446 react with only about $10 \%$ of G-19-positive NFTs.

On the other hand, mAb 7F3 does not react with NFTs. The observed differences in immunoreactivity of NFTs with antibodies and antisera against different sequences of DYRK1A suggest that epitopes detected by these antibodies/antisera are masked in complexes of DYRK1A with tau and other proteins in intracellular NFTs.

\section{Nuclear substrates phosphorylated by DYRK1A}

We documented that DYRK1A phosphorylates alternative splicing factor (ASF) at three sites (unpublished data). 

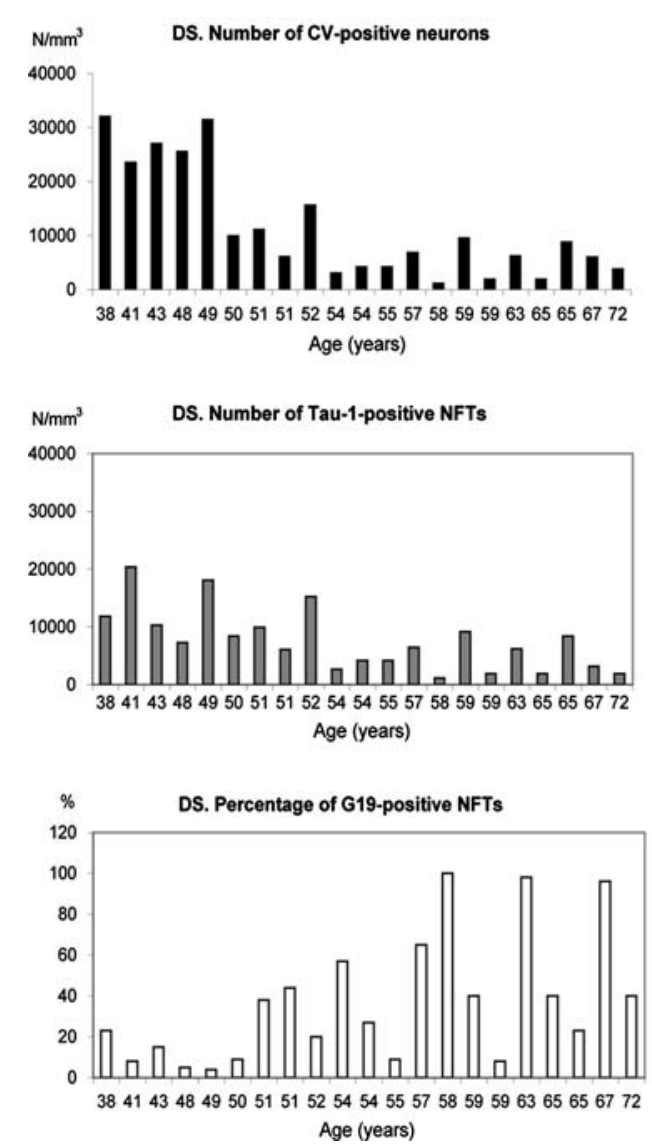

Fig. 6 Graphs illustrate changes in the numerical density of neurons stained with CV and NFTs immunoreactive with Tau-1 antibody and G19-antibody detecting DYRK1A in NFTs in DS subjects (left column) and in $\mathrm{AD}$ (right column) in the second layer of the entorhinal cortex. The early onset of neurofibrillary degeneration results in neuronal loss. In the DS cohort, the decrease in the numerical density of CV-positive neurons and Tau-1-positive NFTs correlates with age $(r=-0.75$ and -0.65 , respectively; $P<0.05)$. DYRK1A-positive NFTs (G19-immunoreactive NFTs) are present in the entorhinal cortex of all DS subjects from 38 to 72 years. The percentage of DYRK1Apositive NFTs (tangles immunoreactive with G19) increases with age

Phosphorylation of ASF by SRPK1 in cytosol results in ASF relocation to the nucleus, whereas phosphorylation by Clk/Sty releases ASF from speckles [32, 45] and recruits it into nascent transcripts, where ASF regulates alternative splicing. Phosphorylation of nuclear ASF by DYRK1A drives ASF back to speckles, decreases involvement in regulation of alternative splicing, and inhibits ASF's ability to promote tau exon 10 inclusion. The result is an increase in $3 \mathrm{R}$ tau level and an imbalance of $3 \mathrm{R} / 4 \mathrm{R}$ tau ratio. The imbalance could be the factor initiating and accelerating neurofibrillary degeneration in the brain of patients with DS.

\section{DYRK1A in NFTs}

Co-localization of DYRK1A with NFTs, described in this article and by in vitro studies showing tau protein
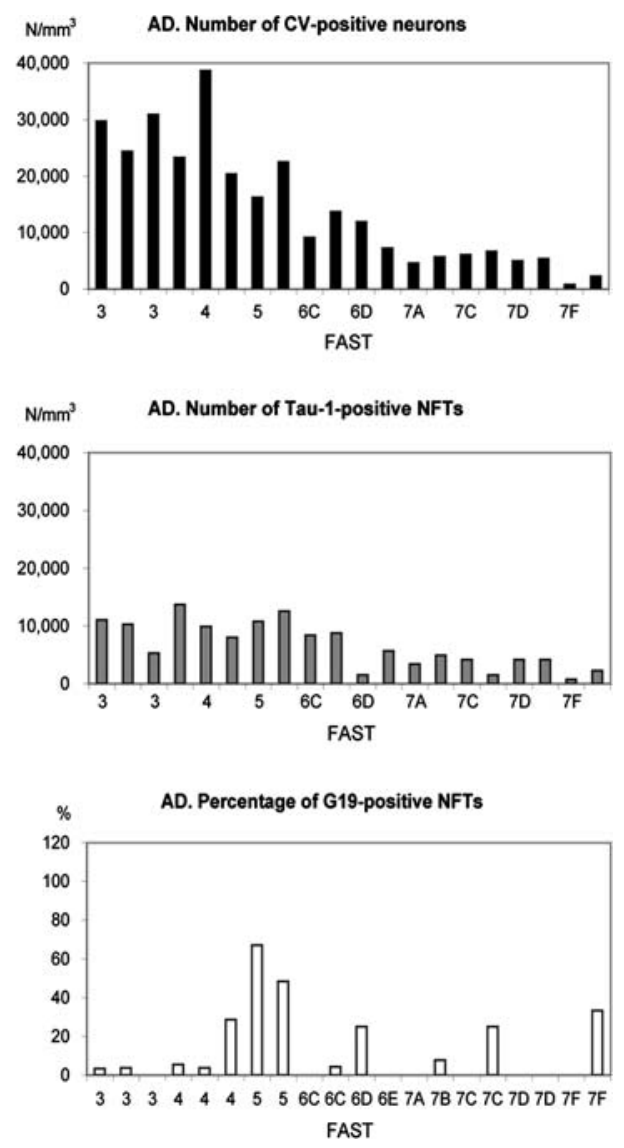

$(r=0.51$; Tau-1-positive NFTs were considered as 100\%). Also in the $\mathrm{AD}$ cohort, a decrease in the numerical density of $\mathrm{CV}$-positive and Tau-1-positive NFTs strongly correlates with stage of disease (FAST; $r=-0.87$, and $r=-0.76$, respectively). However, in contrast to the DS cohort, $40 \%$ of subjects with sporadic AD do not have DYRK1Apositive NFTs; in $30 \%$ of subjects, less than $10 \%$ of Tau-1-positive NFTs are G-19-immunoreactive, and only $30 \%$ of subjects have from 25 to $67 \%$ of G19-immunoreactive NFTs. The majority of subjects with severe sporadic AD (FAST 6 and 7) are free of DYRK1A-positive NFTs

phosphorylation with DYRK1A priming tau phosphorylation with GSK- $3 \beta$, suggests that DYRK1A plays a role in neurofibrillary degeneration and that this process is much more pronounced in neurons of patients with DS than in patients with sporadic AD. In NFTs, tau protein is phosphorylated by several protein kinases, including GSK-3 $\beta$, cyclin-dependent kinase 5 (cdk5), JNK, ERK1/2, and p38 at more than 30 phosphorylation sites $[18,22,34,42,52$, 60]. Several kinases in their activated forms co-localize with NFTs in $\mathrm{AD}$, including mitogen-activated protein kinase (MAPK) [66], microtubule-affinity regulating kinase (MARK) [9], GSK-3 $\beta$ [36, 47, 48, 76], cdk 5 [76], cdc2related kinase [38], and casein kinase $1 \delta$ (Cki $\delta$ ) [57]. This study shows that DYRK1A colocalizes with NFTs in all subjects with DS/AD but in only $60 \%$ of subjects diagnosed with sporadic AD. 
Fig. 7 In a 67-year-old subject with DS, in some cored plaques, diffuse immunoreactivity with G-19, X1079, and 324446 appears in the activated astrocyte body and processes (arrowheads, CAl a-c). In about of $20 \%$ of cored plaques, antibodies to DYRK1A label dystrophic neuronal processes (arrows; d-f). In the CA1 sector, granules (arrowheads) in neurons with granulovacuolar degeneration are negative when stained with antibodies to the $\mathrm{N}$-terminus of DYRK1A ( $7 F 3$, not shown;

$G-19$ shown in $\mathbf{g}$ ) but positive when stained with X1079 and 324446 (h, i). Strong immunoreactivity with all three antibodies appears in corpora amylacea (arrowheads) in the hippocampus (j and $\mathbf{l}$ dentate gyrus; $\mathbf{k}$ CA4). Magnification a-f $\times 210$, $\mathbf{g}-\mathbf{i} \times 1,075, \mathbf{j}-\mathbf{l} \times 210$
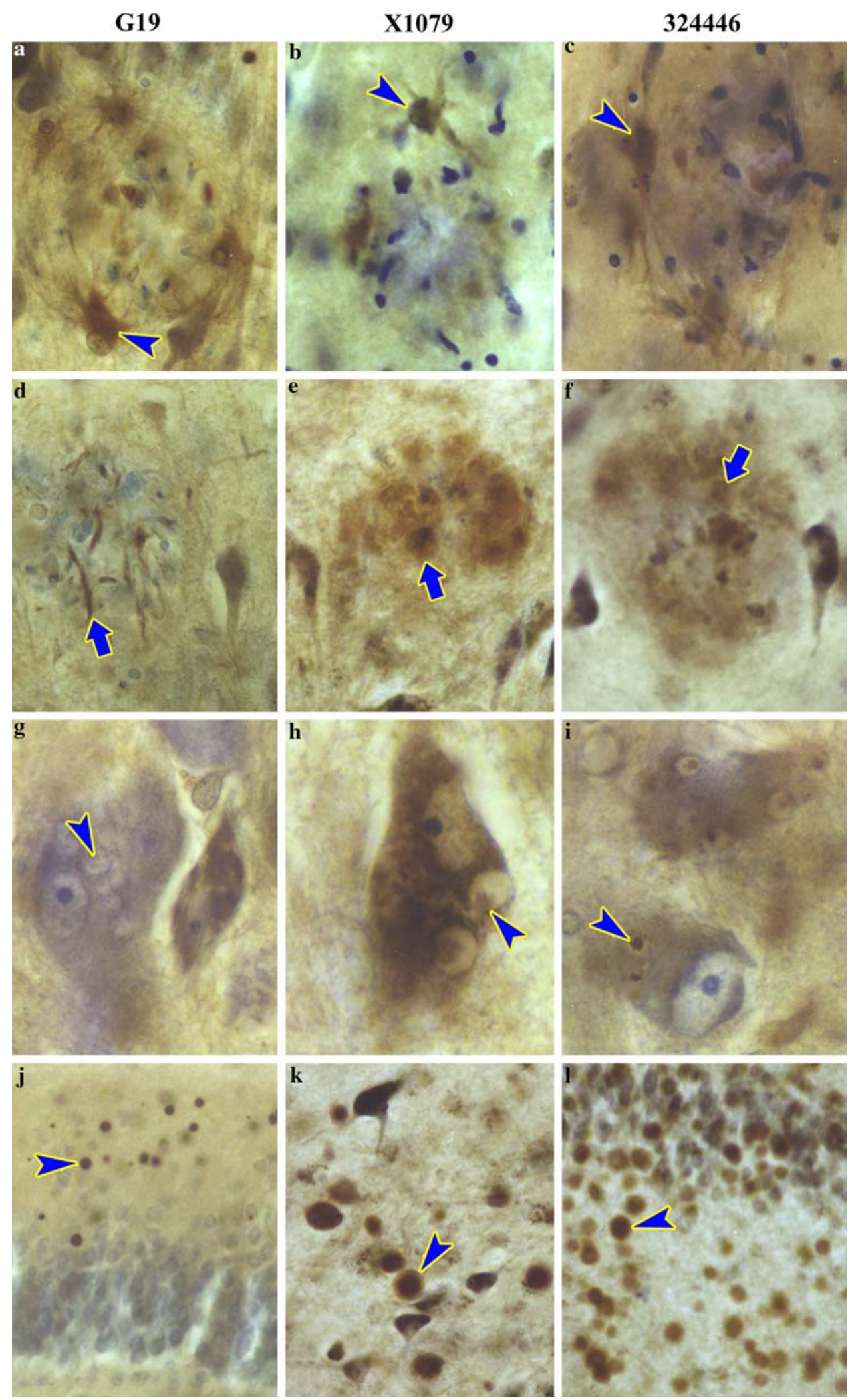

in fetuses with DS show reduced (HACS1) or unmodified levels of some proteins (alpha A-crystalline, FTCD, GARS-AIRS-GART, and CBS), including DYRK1A, which are encoded by genes on chromosome 21 . These data
A gene dosage effect has been proposed to explain the role of an extra chromosome 21 in the pathology of DS. Studies 
call into question the link between overexpressed genes and the formation of DS phenotype in utero [8].

However, in adults with DS, Western blots revealed an almost $50 \%$ higher level of DYRK1A protein in extracts from the frontal cortex. The study of other cortical regions in the human cortex confirmed a $50 \%$ increase in expression of this kinase linked to an extra copy of DyrklA gene in subjects with trisomy of chromosome 21 and DS [13]. The presence of DYRK1A-positive NFTs in all subjects with DS/AD but in only $60 \%$ in sporadic AD cases, and a much higher percentage of NFTs in DS subjects than in subjects with sporadic AD appears to reflect the overexpression of the DYRK1A gene and the link between the excessive amount of this kinase and the onset and progression of neurofibrillary degeneration. Overexpression of the APP gene, increased concentrations of $\mathrm{A} \beta$ in DS fetuses [64], and the early onset of diffuse $[35,73]$ and fibrillar $\mathrm{A} \beta$ deposits [73] represent additional evidence of the selective overexpression of genes throughout the entire lifespan of patients with DS.

\section{Differences in NFTs' immunoreactivity in DS/AD and sporadic $\mathrm{AD}$}

GSK3 $\beta$, cyclin-dependent kinase (cdk) 5 [76], and DYRK1A were found in only some NFTs. These observations and the significant differences in NFTs' immunoreactivity in patients with DS/AD and sporadic AD imply the presence of multiple patterns of tau phosphorylation and neurofibrillary degeneration. Perhaps, the percentage of NFTs that are positive to a given kinase is disease-, stage of disease-, and brain structure-specific. The present observations may also suggest that patterns of tau phosphorylation with DYRK1A in persons with DS are different than those in patients with sporadic AD. This difference may reflect a variation in the progression of neurofibrillary degeneration and, possibly, neuronal loss. In spite of the presence of significant neurofibrillary degeneration and amyloidosis- $\beta$ in almost all patients with DS 40 years of age and older [72, 73], clinically significant functional deterioration has not been reported to increase in prevalence until some 10 years later [24, 33, 76]. The prevalence of dementia in the DS cohort is estimated as 55\% in the age group of 50-59 years, and $75 \%$ in subjects older than 60 years of age [33]. One may hypothesize that tau phosphorylation and neurofibrillary degeneration are modified in persons with DS/AD when compared to sporadic AD. The presence of DYRK1A in the NFTs of the youngest DS subjects with neurofibrillary degeneration indicates DYRK1A's contribution to the early onset of NFTs in DS. A lower percentage of G-19positive NFTs in DS subjects before they reach 50 years of age and a high percentage in subjects in their 50 s or 60 s might be an indication of an enhanced contribution of
DYRK1A to tau phosphorylation and neurofibrillary degeneration in older patients with DS. The lack of immunoreactivity of NFTs with antiserum detecting DYRK1A (G19) in $40 \%$ of AD subjects and the low percentage of G19-positive NFTs in the other $30 \%$ of AD subjects reflect the difference in contribution of overexpressed DYRK1A to neurofibrillary degeneration in DS/AD and the contribution of normal level of DYRK1A in sporadic AD.

The study of chromosome 21 genetic associations with late-onset AD revealed that the DYRK1A gene has the highest significance as a genetic risk factor [30]. The dramatic difference between DYRK1A's role in neurofibrillary degeneration in DS/AD and in sporadic AD appears to be the product of several molecular mechanisms, including (a) the cytoplasmic pathway with phosphorylation of tau by DYRK1A and the priming of tau phosphorylation by GSK3b [40, 78], (b) the nuclear pathway with phosphorylation of ASF by DYRK1A and the imbalance between 3R and 4R tau (unpublished data), as well as (c) phosphorylation of APP by DYRK1A at $\mathrm{Thr}^{668}$ in vitro and in mammalian cells [54]. The experiments showing that $\mathrm{A} \beta$ loading in neuroblastoma cells and transgenic mice increases expression of DYRK1A gene, resulting in hyperphosphorylation of tau at $\mathrm{Thr}^{212}$, support the hypothesis that DYRK1A could be a key molecule linking $\beta$-amyloid production with tau phosphorylation [30, 53, 54].

DYRK1A in neuropil threads and dystrophic neurites, but not in ghost tangles

A striking feature of the immunolabeling of neurons with neurofibrillary degeneration with antibodies to GSK-3 $\beta$, MPM-2, and cdc2 is the positive reaction with PHFs in the neuronal body but the only sparse reactivity with neuropil threads and dystrophic neurites in the plaque perimeter [67, 76]. It was proposed that this difference might be attributable to a differential sensitivity of neuritic components to fixation [38]. The strong immunostaining of NFTs observed in the cell body in the majority of neurons with neurofibrillary degeneration in older subjects with DS/AD, the absence of immunoreactivity with antiserum G-19 in neuronal processes (neuropil threads), and the sparse immunoreactivity observed in dystrophic neurites in cored plaque corona may indicate that DYRK1A is involved in tau phosphorylation mainly in the cell body. These observations may also suggest that the pattern of tau phosphorylation in the cell body is different from that in the dendrites, and that G-19-positive complexes of abnormally phosphorylated tau do not extend to dendrites. However, the possibility that the lack of reaction with antibodies against DYRK1A in neurofibrillary threads in dendrites and synapses could be the result of masking of epitopes in vivo or the loss of immunoreactivity postmortem including tissue processing cannot be excluded at this phase of study. 
Staining of intracellular NFTs with antibodies to cdc and MPM-2 was considered evidence that these kinases play a role in the early stages of neurofibrillary degeneration [67]. Transformation of intracellular NFTs into extracellular ghost tangles is associated with the loss of epitopes recognized by antibodies raised against the amino- and carboxytermini of tau [4]. Extracellular NFTs are immunonegative not only to DYRK1A but also to GSK-3 [76]. The majority of ghost tangles are also negative when stained with antibodies against cdc2 and MPM-2 [67]. Proteolytic processing with a loss of the $\mathrm{N}$ - and $\mathrm{C}$-terminal portions of tau is associated with the secondary deposition on NFTs of ubiquitin [3, 63], of heparan sulfate proteoglycans [3], and, in some brain regions, of $\mathrm{A} \beta[4,63,71,75]$. The absence of DYRK1A in ghost tangles indicates that this binding reflects a contribution to neurofibrillary degeneration rather than unspecific adhesion to already formed NFTs.

\section{Lack of DYRK1A in NFTs in aged control subjects}

In general, the properties of NFTs in aged individuals without dementia recapitulate those found in NFTs in AD [10]. In contrast to the accumulation of DYRK1A in about $50 \%$ of NFTs in DS subjects and the much lower percentage of NFTs in patients with sporadic AD, NFTs in control subjects are free of DYRK1A. The lack of DYRK1A in NFTs of aged controls is not due to the absence of this kinase, because immunocytochemistry shows widespread distribution of DYRK1A in neurons. This pattern resembles the accumulation of protein kinase $\mathrm{M} \zeta(\mathrm{PKM} \zeta)$ in the NFTs of subjects with AD but the absence in NFTs of aged control subjects [10]. The observed NFT immunoreactivity indicates that the association of kinases with NFTs is kinasespecific and aging-, $\mathrm{AD}-$, and $\mathrm{AD} / \mathrm{DS}$-specific.

\section{DYRK1A in GVD and corpora amylacea}

Granulovacuolar degeneration is observed in a small percentage of neurons in the majority of normal aged subjects, and their number increases in persons with $A D$ [65]. The granular component of vacuoles reacts with antibodies to tubulin [49] and antibodies to abnormally phosphorylated tau [11], and GSK-3 $\beta[36,76]$. The presence of DYRK1A immunoreactivity in granules in neurons with GVD detected with C-terminal antibodies and the lack of reactivity with antibodies against the $\mathrm{N}$-terminus may indicate that only N-terminally truncated products of DYRK1A processing contribute to GVD or selectively accumulate in these granules.

Corpora amylacea are considered as a sign of the earliest changes associated with aging and/or neurodegeneration in neurons [6], including axons [1, 7] and synapses [2] as well as glial cells, especially astrocytes $[6,12,56,61]$. Strong immunoreactivity with antibody 7F3 [70] and with antibodies G-19, X1073, and 324446 suggests the involvement of DYRK1A in this form of neuron and astrocyte degeneration and the early onset of these changes in DS.

DYRK1A appears to be involved in many forms of neuronal degeneration including Lewy bodies, a pathological hallmark of Parkinson's disease. DYRK1A phosphorylates $\alpha$-synuclein at $\operatorname{Ser}^{87}$ and selectively binds to $\alpha$-synuclein in transformed and primary neuronal cells. The fact that aggregates formed by phosphorylated $\alpha$-synuclein are more toxic when compared with aggregates composed of unmodified wild-type $\alpha$-synuclein, suggests that $\alpha$-synuclein inclusion formation regulated by DYRK1A may affect neuronal cell viability [28].

Phosphorylation of tau protein by one kinase facilitates or inhibits phosphorylation by other protein kinases in vitro $[26,58,68,74,77]$ and in vivo [39]. The reported findings and other studies [40] suggest that kinases work in a coordinated way and that overexpressed DYRK1A is a major factor contributing to the DS-specific pattern of neurodegeneration. DYRK1A's contribution to neurofibrillary degeneration in DS appears to be much more significant than in subjects with two copies of the DYRKIA gene and sporadic AD. The presence of DYRK1A not only in NFTs but also in granules in GVD and in corpora amylacea suggests that DYRK1A is involved in all three forms of degeneration and may contribute to the early onset of these pathologies in DS.

Protein kinase A (PKA) might be involved in the abnormal phosphorylation of tau in an upstream position to GSK3 and possibly other proline-directed protein kinases [39]. DYRK1A phosphorylates tau at several sites and facilitates the phosphorylation of tau at several sites by GSK-3 [40, 74]. Whereas PKA is considered to be a potential therapeutic target for the treatment of neurofibrillary degeneration in patients with sporadic AD [39], overexpressed DYRK1A might be considered a major target for treatment of neuronal degeneration in patients with DS/AD.

Western blots demonstrated increase of DYRK1A in the DS group when compared to the control and AD group. The presence of DYRK1A in NFTs in sporadic AD indicates that DYRK1A contributes to tau phosphorylation even in the absence of increased gene dosage. However, an increase in DYRK1A-positive NFTs in a gene dosagedependent manner in subjects with DS, including the youngest NFT-positive DS subjects, indicates that overexpressed DYRK1A contributes to the early onset of neurofibrillary degeneration in DS. The correlation between the percentage of G19-positive neurons and the age of DS subjects suggests that the contribution of DYRK1A to neurofibrillary degeneration increases in the later stages of $\mathrm{AD}$ in the DS cohort. Immunoreactivity with antibodies against DYRK1A in granules in GVD and in corpora amylacea 
suggests that DYRK1A is involved in the early onset of all three forms of degeneration in DS.

Acknowledgments This study was supported in part by funds from the New York State Office of Mental Retardation and Developmental Disabilities and grants from the National Institutes of Health, National Institute of Child Health and Human Development R01 HDO43960, and the National Institute of Aging, PO1-HD35897, AG03051, AG08051, HD38295, and R01 AG027429; and the Alzheimer's Association IIRG-05-13095. The authors thank Ms. Maureen Marlow for editorial corrections; and Ms. Jadwiga Wegiel, Cathy Wang, and En $\mathrm{Wu}$ Zhang for histology and immunocytochemistry; and Mr. James C.M. Chen and Ms. Heni Hong of IBR's monoclonal antibody core facility for producing antibody 7F3. The tissue was provided by the Aging and Dementia Research Center at New York University, the Brain and Tissue Bank at the New York State Institute for Basic Research in Developmental Disabilities, and the Brain and Tissue Bank for Developmental Disorders at the University of Maryland, Baltimore (supported by NICHD contract \# NO1-HD-4-3368 and N01-HD-43383).

Open Access This article is distributed under the terms of the Creative Commons Attribution Noncommercial License which permits any noncommercial use, distribution, and reproduction in any medium, provided the original author(s) and source are credited.

\section{References}

1. Averback P, Langevin H (1978) Corpora amylacea of the lumbar spinal cord and peripheral nervous system. Arch Neurol 35:95-96

2. Averback P (1981) Parasynaptic corpora amylacea in the striatum. Arch Pathol Lab Med 105:334-335

3. Bancher C, Brunner C, Lassmann H, Budka H, Jellinger K, Wiche $\mathrm{G}$ et al (1989) Accumulation of abnormally phosphorylated tau precedes the formation of neurofibrillary tangles in Alzheimer's disease. Brain Res 477:90-99. doi:10.1016/0006-8993(89)91396-6

4. Bondareff W, Wischik CM, Novak M, Amos WB, Klug A, Roth M (1990) Molecular analysis of neurofibrillary degeneration in Alzheimer's disease: an immunohistochemical study. Am J Pathol 137:711-723

5. Braak H, Braak E (1991) Neuropathological staging of Alzheimerrelated changes. Acta Neuropathol 82:239-259. doi:10.1007/ BF00308809

6. Buervenich S, Olson L, Galtar D (2001) Nestin-like immunoreactivity of corpora amylacea in aged human brain. Brain Res Mol Brain Res 94:204-208. doi:10.1016/S0169-328X(01)00166-8

7. Cavanagh JB (1999) Corpora amylacea and the family of polyglucosan diseases. Brain Res Brain Res Rev 29:265-295. doi:10.1016/S0165-0173(99)00003-X

8. Cheon MS, Bajo M, Kim SH, Claudio JO, Stewart AK, Patterson $D$ et al (2003) Protein levels of genes encoded on chromosome 21 in fetal Down syndrome brain: challenging the gene dosage effect hypothesis. Amino Acids 24:119-125

9. Chin JY, Knowles RB, Schneider A, Drewes D, Mandelkow E-M, Hyman BT (2000) Microtubule-affinity regulating kinase (MARK) is tightly associated with neurofibrillary tangles in Alzheimer brain: a fluorescence resonance energy transfer study. J Neuropathol Exp Neurol 59:966-971

10. Crary JF, Shao CY, Mirra SS, Hernandez AI, Sacktor TC (2006) Atypical protein kinase $\mathrm{C}$ in neurodegenerative disease I: PKM $\zeta$ aggregates with limbic neurofibrillary tangles and AMPA receptors in Alzheimer disease. J Neuropathol Exp Neurol 65:319-326. doi:10.1097/01.jnen.0000218442.07664.04
11. Dickson DW, Ksiezak-Reading H, Davies P, Yen SH (1987) A monoclonal antibody that recognizes a phosphorylated epitope in Alzheimer neurofibrillary tangles, neurofilaments and tau proteins immunostains granulovacuolar degeneration. Acta Neuropathol 73:254-258. doi:10.1007/BF00686619

12. Dinda AK, Sarkar C, Roy S, Kharabanda K (1992) Immunohistochemical, ultrastructural and immunoelectron microscopic study of glial fibrillary acidic protein in corpora amylacea. Indian J Med Res 96:245-249

13. Dowjat WK, Adayev T, Kuchna I, Nowicki K, Palminiello S, Hwang YW et al (2007) Trisomy-driven overexpression of DYRK1A kinase in the brain of subjects with Down syndrome. Neurosci Lett 413:77-81. doi:10.1016/j.neulet.2006.11.026

14. Ferrer I, Barrachina M, Puig B, Martinez de Lagran M, Marti E, Avila J et al (2005) Constitutive Dyrk1A is abnormally expressed in Alzheimer disease, Down syndrome, Pick disease, and related transgenic models. Neurobiol Dis 20:392-400. doi:10.1016/j.nbd. 2005.03.020

15. Frackowiak J, Mazur-Kolecka B, Kaczmarski W, Dickson D (2001) Deposition of Alzheimer's vascular amyloid- $\beta$ is associated with a decreased expression of brain L-3-hydroxyacyl-coenzyme A dehydrogenase (ERAB). Brain Res 907:44-53. doi:10.1016/S0006-8993(01)02497-0

16. Frackowiak J, Miller DL, Potempska A, Sukontasup T, MazurKolecka B (2003) Secretion and accumulation of A $\beta$ by brain vascular smooth muscle cells from AßPP-Swedish transgenic mice. J Neuropathol Exp Neurol 62:685-696

17. Goedert M, Spillantini MG, Jakes R, Rutherford D, Crowther RA (1989) Multiple isoforms of human microtubule-associated protein tau: sequences and localization in neurofibrillary tangles of Alzheimer's disease. Neuron 3:519-526. doi:10.1016/08966273(89)90210-9

18. Gong C-X, Liu F (2005) Grundke-Iqbal 1: post-translational modifications of tau protein in Alzheimer's disease. J Neural Transm 112:813-838. doi:10.1007/s00702-004-0221-0

19. Grundke-Iqbal I, Iqbal K, Tung YC, Quinlan M, Wisniewski HM, Binder LI (1986) Abnormal phosphorylation of the microtubule associated protein $\tau$ (tau) in Alzheimer cytoskeletal pathology. Proc Natl Acad Sci USA 83:4913-4917. doi:10.1073/pnas.83. 13.4913

20. Guimera J, Casas C, Pucharcos C, Solans A, Domenech A, Planas AM et al (1996) A human homologue of Drosophila minibrain $(M N B)$ is expressed in the neuronal regions affected in Down syndrome and maps to the critical region. Hum Mol Genet 9:13051310. doi: $10.1093 / \mathrm{hmg} / 5.9 .1305$

21. Guimera J, Casas C, Estivill X, Pritchard M (1999) Human Minibrain homologue (MNBH/DYRK1): characterization, alternative splicing, differential tissue expression, and overexpression in Down syndrome. Genomics 57:407-418. doi:10.1006/geno. 1999.5775

22. Hanger DP, Betts JC, Loviny TLF, Blackstock WP, Anderton BH (1998) New phosphorylation sites identified in hyperphosphorylated tau (paired helical filament-tau) from Alzheimer's disease brain using nanoelectrospray mass spectrometry. J Neurochem 71:2465-2476

23. Himpel S, Panzer P, Eirmbter K, Czajkowska H, Sayed M, Packman LC et al (2001) Identification of the autophosphorylation sites and characterization of their effects in the protein kinase DYRK1A. Biochem J 359:497-505. doi:10.1042/0264-6021: 3590497

24. Holland AJ, Hon J, Huppert FA, Stevens F, Watson P (1998) Population-based study of the prevalence and presentation of dementia in adults with Down's syndrome. Br J Psychol 172:493-498

25. Iqbal K, Braak H, Braak E, Grundke-Iqbal I (1993) Silver labeling of Alzheimer neurofibrillary changes and brain $\beta$-amyloid. J Histotechnol 16:335-342 
26. Jicha GA, Weaver C, Lane E, Vianna C, Kress Y, Rockwood J et al (1999) cAMP-dependent protein kinase phosphorylations on tau in Alzheimer's disease. J Neurosci 19:7486-7494

27. Kentrup H, Becker W, Heukelbach J, Wilmes A, Scurmann A, Huppertz C et al (1996) Dyrk, a dual specificity protein kinase with unique structural features whose activity is dependent on tyrosine residues between subdomains VII and VIII. J Biol Chem 271:3488-3495. doi:10.1074/jbc.271.7.3488

28. Kim EJ, Sung Y, Lee HJ, Rhim H, Hasegawa M, Iwatsubo T, Min do S, Kim J, Paik SR, Chung KC (2006) Dyrk1A phosphorylates alpha-synuclein and enhances intracellular inclusion formation. J Biol Chem 281:33250-33257. doi:10.1074/jbc.M606147200

29. Kim KS, Miller DL, Sapienza VJ, Chen CMJ, Bai C, GrundkeIqbal I et al (1988) Production and characterization of monoclonal antibodies reactive to synthetic cerebrovascular amyloid peptide. Neurosci Res Commun 2:121-130

30. Kimura R, Kamino K, Yamamoto M, Nuripa A, Kida T, Kazui H et al (2007) The DYRK1A gene, encoded in chromosome 21 Down syndrome critical region, bridges between beta-amyloid production and tau phosphorylation in Alzheimer disease. Hum Mol Genet 16:15-23. doi:10.1093/hmg/ddl437

31. Kitamoto T, Ogomori K, Tateishi J, Prusiner S (1987) Methods in laboratory investigation: formic acid pretreatment enhances immunostaining of cerebral and systemic amyloids. Lab Invest 57:230-236

32. Koizumi J, Okamoto Y, Onogi H, Mayeda A, Krainer AR, Hagiwara M (1999) The subcellular localization of ASF is regulated by direct interaction with SR protein kinases (SRPKs). J Biol Chem 274:11125-11131. doi:10.1074/jbc.274.16.11125

33. Lai F, Williams RS (1989) A prospective study of Alzheimer disease in Down syndrome. Arch Neurol 46:849-858

34. Lee VM-Y, Goedert M, Trojanowski JQ (2001) Neurodegenerative tauopathies. Annu Rev Neurosci 24:1121-1159. doi:10.1146/ annurev.neuro.24.1.1121

35. Lemere CA, Blusztajn JK, Yamaguchi H, Wisniewski T, Saido TC, Selkoe DJ (1996) Sequence of deposition of heterogenous amyloid $\beta$-peptides and APO E in Down syndrome: implications for initial events in amyloid plaque formation. Neurobiol Dis 3:16-32. doi:10.1006/nbdi.1996.0003

36. Leroy K, Boutajangout A, Authelet M, Woodgett JR, Anderton BA, Brion J-P (2002) The active form of glycogen synthase kinase- $3 \beta$ is associated with granulovacuolar degeneration in neurons in Alzheimer's disease. Acta Neuropathol 103:91-99. doi:10.1007/s004010100435

37. Leverenz J, Raskind MA (1998) Early amyloid deposition in the medial temporal lobe of young Down syndrome patients: a regional quantitative analysis. Exp Neurol 150:296-304. doi:10.1006/ exnr.1997.6777

38. Liu W-K, Williams RT, Hall FL, Dickson DW, Yen S-H (1995) Detection of a Cdc2-related kinase associated with Alzheimer paired helical filaments. Am J Pathol 146:228-238

39. Liu SJ, Zhang JY, Li HL, Fang ZY, Wang Q, Deng HM et al (2004) Tau becomes a more favorable substrate for GSK-3 when it is prephosphorylated by PKA in rat brain. J Biol Chem 279:50078-50088. doi:10.1074/jbc.M406109200

40. Liu F, Liang Z, Wegiel J, Hwang Y-W, Iqbal K, Grundke-Iqubal I, et al. (2008) Over-expression of Mnb/Dyrk1A contributes to neurofibrillary degeneration in Down syndrome. FASEB J 22: (in press)

41. Lott IT, Lai F (1982) Dementia in Down's syndrome: observations from a neurologic clinic. Appl Res Ment Retard 3:233-239. doi:10.1016/0270-3092(82)90017-0

42. Lovestone S, Reynolds CH (1997) The phosphorylation of tau: a critical stage in neurodevelopment and neurodegenerative processes. Neuroscience 78:309-324. doi:10.1016/S0306-4522(96) $00577-5$
43. Morishima-Kawashima M, Hasegawa M, Takio K, Suzuki M, Yoshida H, Titani K et al (1995) Proline directed and non-proline directed phosphorylation of PHF-tau. J Biol Chem 270:823-829. doi:10.1074/jbc.270.2.823

44. The National Institute on Aging and Reagan Institute Working Group on Diagnostic Criteria for the Neuropathological Assessment of Alzheimer's Disease (1997) Consensus recommendations for the postmortem diagnosis of Alzheimer's disease. Neurobiol Aging 18:1-2. doi:10.1016/S0197-4580(96)00213-8

45. Ngo JCK, Chakrabarti S, Ding J-H, Velazques-Dones A, Nolen B, Aubol BE et al (2005) Interplay between SRPK and Clk/Sty kinases in phosphorylation of the splicing factor ASF/SF2 is regulated by a docking motif in ASF/SF2. Mol Cell 20:77-89. doi:10.1016/ j.molcel.2005.08.025

46. Okui M, Ide T, Morita K, Funakoshi E, Ito F, Ogita K et al (1999) High-level expression of the Mnb/Dyrk1A gene in brain and heart during rat early development. Genomics 62:165-171. doi:10.1006/ geno.1999.5998

47. Pei J-J, Tanaka T, Tung Y-C, Braak E, Iqbal K, Grundke-Iqbal I (1997) Distribution, levels, and activity of glycogen synthase kinase-3 in the Alzheimer disease brain. J Neuropathol Exp Neurol 57:70-78. doi:10.1097/00005072-199701000-00007

48. Pei J-J, Braak E, Braak H, Grundke-Iqbal I, Iqbal K, Winblad B et al (1999) Distribution of active glycogen synthase kinase $3 \beta$ (GSK-3 $\beta)$ in brains staged for Alzheimer disease neurofibrillary changes. J Neuropathol Exp Neurol 58:1010-1019. doi:10.1097/ 00005072-199909000-00011

49. Price DL, Altschuler RJ, Struble RG, Casanova MF, Cork LC, Murphy DB (1986) Sequestration of tubulin in neurons in Alzheimer's disease. Brain Res 385:305-310. doi:10.1016/00068993(86)91077-2

50. Rahmani Z, Blouin J, Creaau-Goldberg N, Watkins PC, Mattei J, Poissonier $\mathrm{M}$ et al (1989) Critical role of the D21S55 region on chromosome 21 in the pathogenesis of Down syndrome. Proc Natl Acad Sci USA 86:5958-5962. doi:10.1073/pnas.86.15.5958

51. Reisberg B (1988) Functional assessment staging (FAST). Psychopharmacol Bull 24:653-659

52. Reynolds $\mathrm{CH}$, Betts JC, Blackstock WP, Nebreda AR, Anderton BH (2000) Phosphorylation sites on tau identified by nanoelectrospray mass spectrometry: differences in vitro between the mitogen activated protein kinases ERK2, c-Jun N-terminal kinase and P38, and glycogen synthase kinase-3 $\beta$. J Neurochem 74:1587-1595. doi:10.1046/j.1471-4159.2000.0741587.x

53. Ryoo SR, Jeong HK, Radnaabazar C, Too JJ, Cho HJ, Lee HW et al (2007) DYRK1A-mediated hyperphosphorylation of Tau. A functional link between Down syndrome and Alzheimer disease. J Biol Chem 2828:34850-34857. doi:10.1074/jbc.M707358200

54. Ryoo SR, Cho HJ, Le HW, Jeong HK, Radnaabazar C, Kim YS et al (2008) Dual specificity tyrosine (Y)-phosphorylation regulated kinase 1A-mediated phosphorylation of amyloid precursor protein: evidence for a functional link between Down syndrome and Alzheimer's disease. J Neurochem 104:1333-1344. doi:10.1111/ j.1471-4159.2007.05075.x

55. Sadowski M, Wisniewski HM, Tarnawski M, Kozlowski P, Lach B, Wegiel J (1999) Neuronal loss in the entorhinal cortex of aged subjects with Down syndrome. Acta Neuropathol 97:156-164. doi:10.1007/s004010050968

56. Sbarbati A, Carner M, Colletti V, Osculati F (1996) Extrusion of corpora amylacea from the marginal glia at the vestibular root entry zone. J Neuropathol Exp Neurol 55:196-201. doi:10.1097/ 00005072-199602000-00008

57. Schwab C, DeMaggio AJ, Ghoshal N, Binder LI, Kuret J, McGeer PL (2000) Casein kinase 1 delta is associated with pathological accumulation of tau in several neurodegenerative diseases. Neurobiol Aging 21:503-510. doi:10.1016/S0197-4580(00) 00110-X 
58. Sengupta A, Kabat J, Novak M, Wu Q, Grundke-Iqbal I, Iqbal K (2000) Phosphorylation of tau at both Thr 231 and Ser 262 is required for maximal inhibition of its binding to microtubules. Arch Biochem Biophys 357:299-309. doi:10.1006/abbi.1998.0813

59. Shindoh N, Kudoh J, Maeda H, Yamaki A, Minoshima S, Shimizu Y et al (1996) Cloning of a human homolog of the Drosophila minibrain/rat Dyrk gene from the Down syndrome critical region of chromosome 21. Biochem Biophys Res Commun 225:92-99. doi:10.1006/bbrc. 1996.1135

60. Singh TJ, Zaidi T, Grundke-Iqbal I, Iqbal K (1996) Modulation of GSK-3-catalyzed phosphorylation of microtubule-associated protein tau by non-proline-dependent protein kinases. FEBS Lett 358:4-8. doi:10.1016/0014-5793(94)01383-C

61. Singhrao SK, Neal JW, Piddlesden SJ, Newman GR (1994) New immunocytochemical evidence for a neuronal/oligodendroglial origin for corpora amylacea. Neuropathol Appl Neurobiol 20:6673. doi:10.1111/j.1365-2990.1994.tb00958.x

62. Song W-J, Sternberg LR, Kasten-Sportes C, Van Keuren ML, Chung S-H, Slack AC et al (1996) Isolation of human and murine homologues of the Drosophila minibrain gene: human homolog maps to 21q22.2 in the Down syndrome critical region. Genomics 38:331-339. doi:10.1006/geno.1996.0636

63. Tabaton M, Cammarata S, Mancardi G, Manetto V, AutilioGambetti L, Perry G et al (1991) Ultrastructural localization of b-amyloid, $\tau$, and ubiquitin epitopes in extracellular neurofibrillary tangles. Proc Natl Acad Sci USA 88:2098-2102. doi:10.1073/ pnas.88.6.2098

64. Teller JK, Russo C, DeBusk LM, Angelini G, Zaccheo D, DagnaBricarelli F et al (1996) Presence of soluble amyloid B-peptide precedes amyloid plaque formation in Down's syndrome. Nat Med 2:93-95. doi:10.1038/nm0196-93

65. Tomlinson BE, Kitchener D (1972) Granulovacuolar degeneration of hippocampal pyramidal cells. J Pathol 106:165-185. doi:10.1002/ path. 1711060305

66. Trojanowski JQ, Mawal-Dewan M, Schmidt ML, Martin J, Lee VM (1993) Localization of the mitogen-activated protein kinase ERK in Alzheimer's disease neurofibrillary tangles and senile plaque neurites. Brain Res 618:333-337. doi:10.1016/00068993(93)91286-2

67. Vincent I, Jicha G, Rosado M, Dickson DW (1997) Aberrant expression of mitotic cdc2/cyclin B1 kinase in degenerating neurons of Alzheimer's disease brain. J Neurosci 17:3588-3598

68. Wang J, Kudoh J, Shinatni A, Minoshima S, Schimizu N (1998) Identification of two novel 5' noncoding exons in human $M N B /$ DYRK gene and alternatively spliced transcripts. Biochem Biophys Res Commun 250:704-710. doi:10.1006/bbrc.1998.9392
69. Wegiel J, Wisniewski HM, Dziewiatkowski J, Popovitch ER, Tarnawski M (1996) Differential susceptibility to neurofibrillary pathology among patients with Down syndrome. Dementia 7:135141. doi: $10.1159 / 000106868$

70. Wegiel J, Kuchna I, Nowicki K, Frackowiak J, Dowjat K, Silverman WP et al (2004) Cell type- and brain structure-specific patterns of distribution of minibrain kinase in human brain. Brain Res 1010:69-80. doi:10.1016/j.brainres.2004.03.008

71. Wegiel J, Kuchna I, Nowicki K, Imaki H, Wegiel J, Mehta P et al (2007) Intraneuronal $A \beta$ immunoreactivity is not a predictor of brain amyloidosis- $\beta$ or neurofibrillary degeneration. Acta Neuropathol 113:389-402. doi:10.1007/s00401-006-0191-4

72. Wisniewski KE, Wisniewski HM, Wen GY (1985) Occurrence of neuropathologic changes and dementia of Alzheimer's disease in Down's syndrome. Ann Neurol 17:278-282. doi:10.1002/ana. 410170310

73. Wisniewski HM, Wegiel J, Popovitch ER (1994) Age-associated development of diffuse and thioflavin-S-positive plaques in Down syndrome. Dev Brain Dysfunct 7:330-339

74. Woods YL, Cohen P, Becker W, Jakes R, Goedert M, Wang X et al (2001) The kinase DYRK phosphorylates protein-synthesis initiation factor $\operatorname{elF} 2 \mathrm{~B} \varepsilon$ at Ser539 and the microtubule-associated protein tau at Thr212: potential role for DYRK as a glycogen synthase kinase 3-priming kinase. Biochem J 355:609-615

75. Yamaguchi H, Nakazato Y, Shoji M, Okamoto K, Ihara Y, Morimatsu M et al (1991) Secondary deposition of beta-amyloid within extracellular neurofibrillary tangles in Alzheimer-type dementia. Am J Pathol 138:699-705

76. Yamaguchi $\mathrm{H}$, Ishiguro $\mathrm{K}$, Uchida $\mathrm{T}$, Takashima A, Lemere $\mathrm{CA}$, Imahori K (1996) Preferential labeling of Alzheimer neurofibrillary tangles with antisera for tau protein kinase (TPK) I/glycogen synthase kinase- $3 \beta$ and cyclin-dependent kinase 5 , a component of TPK II. Acta Neuropathol 92:232-241. doi:10.1007/s0040 10050513

77. Zheng-Fischhofer Q, Biernat J, Mandelkow EM, Illenberger S, Godemann R, Mandelkow E (1998) Sequential phosphorylation of Tau by glycogen synthase kinase- $3 \beta$ and protein kinase $A$ at Thr212 ans Ser214 generates the Alzheimer-specific epitope of antibody AT100 and requires a paired-helical-filament-like conformation. Eur J Biochem 252:542-555. doi:10.1046/j.14321327.1998.2520542.x

78. Zigman WB, Schupf N, Sersen E, Silverman W (1995) Prevalence of dementia in adults with and without Down syndrome. Am J Ment Retard 100:403-412 